\title{
A FUBINI THEOREM FOR ITERATED STOCHASTIC INTEGRALS
} BY MARC A. BERGER ${ }^{1}$ AND VICTOR J. MIZEL ${ }^{2}$

Communicated by D. W. Stroock, July 8, 1977

Let $x(t)$ and $y(t)$ be continuous martingales on the probability space $(\Omega, F, \mathbf{P})$. Consider a bounded region $D$ in $\mathbf{R}_{+}^{2}$ with smooth boundary $\Gamma$. Define $\Gamma_{i}$ to be the portion of $\Gamma$ along which the outward normal points in the direction of quadrant $i(i=1,2,3,4)$. Let $f(t, s)$ be a smooth function on $\bar{D}$. It is desired to evaluate

$$
\iint_{D} f(t, s) d x(s) d y(t) \text { and } \iint_{D} f(t, s) d y(t) d x(s)
$$

by Riemann sums in an Ito-belated fashion. The difference between these sums is then of the form

$$
\pm \Sigma f\left(t_{i}^{\prime}, s_{i}^{\prime}\right)\left[x\left(s_{i+1}^{\prime}\right)-x\left(s_{i}^{\prime}\right)\right]\left[y\left(t_{i+1}^{\prime}\right)-y\left(t_{i}^{\prime}\right)\right]
$$

where $\left(t_{i}^{\prime}, s_{i}^{\prime}\right)$ are points near $\Gamma_{2}$ and $\Gamma_{4}$. Under suitable conditions on $D$ this sum tends to an integral of $f$ along these portions of $\Gamma$. These considerations lead to the following

Theorem (The Correction Formula). Let $(x(t), y(t))$ be a joint martingale. Then

$$
\begin{aligned}
\iint_{D} f(t, s) d x(s) d y(t)+\int_{\Gamma_{2} \cap l} f(t, t) d\langle x, y\rangle(t) \\
=\iint_{D} f(t, s) d y(t) d x(s)+\int_{\Gamma_{4} \cap l} f(t, t) d\langle x, y\rangle(t)
\end{aligned}
$$

where $\langle x, y\rangle$ is the quadratic covariation process of $x$ and $y$, and $l$ is the line $s=t$.

It is to be noted that the rigorous justification of the Correction Formula entails the development of a new type of stochastic integral $I(t)=\int_{t_{0}}^{t} g(t, s) d x(s)$ where $g(t, s)$ is measurable with respect to the sigma-field generated by $\{x(u)-$ $x(s): s \leqslant u \leqslant t$. Conditions are given which insure the existence of $I(t)$ as a limit of Ito-belated Riemann sums. The following result concerning the moments of $I(t)$ is presented.

AMS (MOS) subject classifications (1970). Primary 60H20; Secondary 45D05.

Key words and phrases. Brownian motion, martingale, stochastic integral.

1 Supported by the Fannie and John Hertz Foundation Fellowship Fund.

2 Supported by National Science Foundation Grant MCS-71-02776-A05, by a sabbatical grant from Carnegie-Mellon University, and by the Lady Davis Fellowihip Fund. 
THEOREM. Suppose $\mathrm{E}\left(g(t, s) / F_{s}\right)=0$, a.s. where $F_{s}$ is the sigma-field generated by $\left\{x(u): t_{0} \leqslant u \leqslant s\right\}$. Then

$$
\mathbf{E} I(t)=-\int_{t_{0}}^{t} \mathbf{E} \partial_{s} g(t, s) d x(s)
$$

$$
\mathbf{E}|I(t)|^{2}=\int_{t_{0}}^{t} \mathbf{E}|g(t, s)|^{2} d s+\int_{t_{0}}^{t} \int_{t_{0}}^{t} \mathbf{E} \partial_{s} g(t, s) \partial_{r} g(t, r) d x(s) d x(r)
$$

where $\partial_{s}$ represents the s-stochastic differential.

As an application of the Correction Formula, consider the linear Ito-Volterra equation (see Berger [1])

$$
\xi(t)-\int_{t_{0}}^{t} a(t, s) \xi(s) d w(s)-\int_{t_{0}}^{t} b(t, s) \xi(s) d s=F(t) .
$$

Here $w(t)$ is a Brownian motion. Using the Correction Formula the solution can be obtained by a Neumann series, and takes the form

$$
\xi(t)=F(t)+\int_{t_{0}}^{t} r_{a}(t, s) F(s) d v(s)+\int_{t_{0}}^{t} r_{b}(t, s) F(s) d s
$$

where $v(t)=w(t)-\int_{t_{0}}^{t} a(s, s) d s$ and

$$
\begin{gathered}
r_{a}(t, s)=\sum_{n=1}^{\infty} a_{n}(t, s), \quad r_{b}(t, s)=\sum_{n=1}^{\infty} b_{n}(t, s), \\
a_{1}(t, s)=a(t, s), \quad b_{1}(t, s)=b(t, s), \\
a_{n+1}(t, s)=\int_{s}^{t} a(t, r) a_{n}(r, s) d w(r)+\int_{s}^{t} b(t, r) a_{n}(r, s) d r, \\
b_{n+1}(t, s)=\int_{s}^{t} a(t, r) b_{n}(r, s) d w(r)+\int_{s}^{t} b(t, r) b_{n}(r, s) d r ; \quad n=1,2, \ldots .
\end{gathered}
$$

Corollaries of the Correction Formula include a differentiation rule for $z(t)$ $=h(t, x(t))$ where $h(t, a)=\int_{t_{0}}^{t} \psi(s, x(s), t, a) d x(s)$; and a formula for evaluating $\int_{t_{0}}^{t} \varphi(s) H_{n}\left(\int_{s}^{t} \varphi^{2}(r) d r, \int_{s}^{t} \varphi(r) d w(r)\right) d w(s)$ where $H_{n}(t, x)$ is the Hermite polynomial of degree $n$. There is also included a discussion of stochastic integrals $\int_{t_{0}}^{t} x(\lambda(s)) d x(s)$ where $\lambda(s) \geqslant s$.

\section{REFERENCES}

1. M. A. Berger, Stochastic Ito-Volterra equations, Ph.D. Thesis, Pittsburgh, CarnegieMellon University, 1977.

2. P. A. Meyer, Seminaire de Probabilities. X, Lecture Notes in Math., vol. 511, Springer-Verlag, New York, 1976, pp. 321-326.

DEPARTMENT OF MATHEMATICS, CARNEGIE-MELLON UNIVERSITY, PITTSBURGH, PENNSYLVANIA 15213

DEPARTMENT OF MATHEMATICS, TECHNION, HAIFA, ISRAEL 